\title{
Ästhetische Chirurgie bei Kindern und Jugendlichen?
}

\section{Aesthetic Surgery in Children and Adolescents}

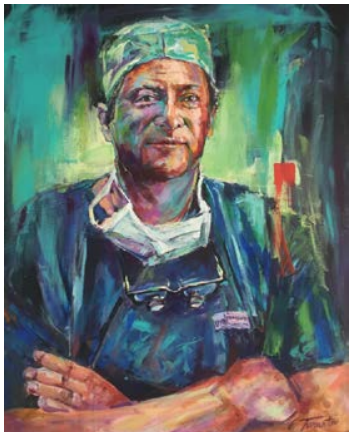

Univ.-Prof. Dr. med. Riccardo E. Giunta
Bibliografie

DOI http://dx.doi.org/

10.1055/s-0041-108318

Handchir Mikrochir Plast Chir

2015; 47: 341-342

(c) Georg Thieme Verlag KG

Stuttgart · New York

ISSN 0722-1819

Korrespondenzadresse

Univ.-Prof. Dr. med.

Riccardo E. Giunta

Handchirurgie, Plastische

Chirurgie und Ästhetische

Chirurgie

Klinikum der Ludwig-

Maximilians

Universität (LMU) München

Pettenkoferstraße 8a

80336 München

r.giunta@med.uni-muenchen.de
Liebe Leserinnen und Leser,

seit Jahrhunderten bemühen sich Plastische Chirurgen durch Verpflanzung, Verlagerung oder den Ersatz von Gewebe um Heilung, Rekonstruktion oder Wiederherstellung nach Verletzung, Tumor, Krankheit oder bei angeborenen Fehlbildungen. Immer wieder sind sie auch in Grenzbereichen tätig. Einige sind umstritten. Sie verlangen daher eine offene Diskussion. Der Schwerpunkt des vorliegenden Hefts will dazu anregen. Einer der Grenzbereiche sind die nicht im traditionellen Sinn der Medizin indizierten Eingriffe bei Kindern und Jugendlichen, um deren äußeres Erscheinungsbild zu korrigieren oder zu verbessern. In keinem anderen chirurgischen Fach werden die Erfolge, aber auch die Misserfolge von operativen Eingriffen so unmittelbar erkannt wie in der Ästhetischen Plastischen Chirurgie, die ausschließlich auf eine Veränderung der sichtbaren Oberfläche des Körpers zielt. Auch deshalb wird in diesem Bereich die Erfüllung der Wünsche des Patienten (oder seiner Umgebung) vom Operateur nachdrücklicher eingefordert als in anderen chirurgischen Fächern. Im besten Interesse des Patienten dagegen Widerstand zu leisten, kann Pflicht und ärztliche Verantwortung sein.

Wenn die Plastische Chirurgie auch in diesem Bereich ärztliches Handeln bleiben und nicht bloße Dienstleistung werden soll, wenn sie also den Patienten nicht zum bloßen Kunden degradieren will, bedarf es einer juristischen und psychologischen Wertung, die uns einerseits sagt, was rechtens, erlaubt und zulässig ist und andererseits vermittelt, welchen Erwartungen und Hoffnungen jugendlicher Patienten wir folgen sollen und welchen nicht.

Auf Initiative meines Kollegen Alexander Berghaus von der HNO-Klinik der Ludwig-Maximilians-Universität, München, wurde kürzlich zu dieser Thematik eine Podiumsdiskussion organisiert. Anlass war die 86. Jahresversammlung der Deutschen Gesellschaft für Hals-Nasen-Ohren-Heilkunde in Berlin im Mai dieses Jahres. Nach den Vorträgen hatte sich eine sehr aufschlussreiche Diskussion ergeben. Die Texte von Herrn Rechtsanwalt Dr. Albrecht Wienke aus Köln [1] und vom Kinder- und Jugendpsychiater Herrn Prof. Michael Kölch aus Berlin [2] geben wichtige Standpunkte wider. Sie einzuordnen, anzuwenden und sowohl an der ärztlich ratsamen Absicht und den operationstechnischen Möglichkeiten zu messen, bleibt allerdings unsere Aufgabe als Plastische Chirurgen.
Die häufigsten ästhetischen Operationen bei Kindern und Jugendlichen waren, nach den Erhebungen der Deutschen Gesellschaft der Plastischen, Rekonstruktiven und Ästhetischen Chirurgen (DGPRÄC) im Jahr 2012, Eingriffe zur Korrektur abstehender Ohren (87\%). Sie werden oft schon im Kindergarten- und Vorschulalter ausgeführt. Gefolgt von Korrekturen der Gynäkomastie (4\%). Es handelt sich dabei um Operationen, die zwar mit dem Ziel der Verbesserung der äußeren Erscheinung, durchgeführt werden, aber durch den Druck und den Einfluss der „Peergroup“ des jugendlichen Patienten ärztlich indiziert sein können. In der Gesellschaft wird das erkannt und anerkannt. Junge Menschen in der Adoleszenz sind dem Spott der Umgebung und den eigenen Insuffizienzgefühlen nicht immer gewachsen. Vergleichbares kann auch für Gynäkomastien, Brustasymmetrien, Makromastien oder anderen Störungen der äußeren Erscheinung des Heranwachsenden gelten. Sie sind in vielen Fällen medizinisch angezeigt, vertretbar und sinnvoll.

Im Gegensatz zu den allein ästhetisch motivierten Operationen, die im Kindes- und Jugendalter aus ethischen, moralischen und juristischen Gründen nicht oder zumindest nicht ohne weiteres durchgeführt werden sollen oder dürfen. Z.B., wenn es um die Anwendung von Füllsubstanzen zur Faltentherapie geht oder um Brustvergrößerungen. Auch die Behandlung der Dysmorphophobie kann nicht chirurgisch erfolgen. Nie vertretbar sind Eingriffe an jungen Menschen, mit denen ihre äußere Erscheinung irgendwelchen „Idolen“ wie etwa den so genannten „Barbie-Puppen“ oder auch Sängeridolen wie etwa Justin Bieber angepasst und angenähert werden sollen. Bedauerliche Fälle dieser Art finden sich im Internet.

Die kritische Betrachtung einer beabsichtigten plastisch-chirurgischen Operation bei Kindern oder jungen Patienten muss deren Erziehungsberechtigte, das Therapieteam und natürlich sie selbst einbeziehen. Die Patientengruppe, um die es geht, ist vom Alter her nur bedingt oder gar nicht entscheidungsfähig und hat nicht immer eine konkrete Vorstellung von den Konsequenzen eines Eingriffs. Plastisch-chirurgische Eingriffe sind nicht im selben Maß reversibel wie Schminke, Lippenstift oder Frisur. Auch wenn die einschlägige Reklame in den Medien das unter Verwendung von Adjektiven wie „kosmetisch“, „ästhetisch“ oder mit Begriffen wie „Schönheitsoperationen" manchmal suggeriert. 
In Österreich wurde 2012 sogar ein Gesetz zur „Durchführung von Ästhetischen Behandlungen und Operationen erlassen.“[3] Es definiert rein ästhetische Operationen im wesentlichen durch die fehlende medizinische Indikation und sieht darin „...eine operativ-chirurgische Behandlung zur Herbeiführung einer subjektiv wahrgenommenen Verbesserung des optischen Aussehens... ohne medizinische Indikation.“ ( $\S 3,80$, Bundesgesetzbuch Österreich). Nach $\S 7.1$, ist eine ästhetische Behandlung oder Operation an Personen, die das 16. Lebensjahr noch nicht vollendet haben, nicht ohne weiteres erlaubt. Sie darf an Personen, die das 16. Lebensjahr oder das 18 . Lebensjahr noch nicht vollendet haben, nur durchgeführt werden, wenn die Einwilligung durch die Erziehungsberechtigten nach einer umfassenden und vollständigen ärztlichen Aufklärung schriftlich gegeben wird.

Hinweise zur Unzulässigkeit oder Zulässigkeit ergeben sich auch durch die Klärung der Frage, wer bei Kindern oder Jugendlichen für die Kosten eines plastisch-chirurgischen Eingriffs aufkommt. Bei Vorliegen einer medizinischen Indikation übernehmen die Versicherungsträger in der Regel die Kosten. Bei rein ästhetisch motivierten Operationen trägt der Patient die Behandlungskosten. Manchmal ist das eine schwierige Entscheidung. Dann muss über den Therapieplan unter Mitwirkung aller Beteiligten entschieden werden. Auch unter Zuziehung eines Jugendpsychologen oder Psychiaters.
Als Fazit ergeben sich folgende Schlussfolgerungen:

- Keine Ästhetischen Operationen bei Kindern- und Jugendlichen ohne medizinische Indikation

- Im Zweifel bis zur Volljährigkeit abwarten

- Ästhetische Operationen nur bei vorliegen einer sich daraus ableitenden klaren medizinischen Indikation

- Medizinische Indikationen bei Jugendlichen immer nur mit Einvernehmen des Patienten und beider Eltern einschließlich Unterschrift aller auf der Patientenaufklärung

- Im Zweifel generell hinzuziehen eines Kinder- und Jugendpsychiaters

- Entscheidend ist die individuelle Entscheidungsfindung im Konsens des Therapieteams

München, im November 2015

Riccardo Giunta

\section{Literatur}

1 Wienke A. Was ist erlaubt? - Ästhetische Eingriffe bei Kindern und Jugendlichen. Handchir Mikrochir Plast Chir 2015; 47: 348-352

2 Kölch M, Izat Y. Ästhetisch-plastische Operationen bei Kindern - Sicht der Kinder- und Jugendpsychiatrie und -psychotherapie. Handchir Mikrochir Plast Chir 2015; 47: 343-347

3 Bundesgesetzblatt für die Republik Österreich Jahrgang. 2012, 80. Bundesgesetz: Durchführung von ästhetischen Behandlungen und Operationen sowie Änderung des Ärztegesetzes 1998; http://www. plastischechirurgie.org/fileadmin/user_upload/AesthOP-Gesetz/bg bla_2012_i_80.pdf 